\title{
The Iwasawa $\lambda$-invariants of $\mathbb{Z}_{p}$-extensions of real quadratic fields
}

\author{
by \\ TAkashi Fukuda (Chiba) and Hisao TAYA (Tokyo)
}

1. Introduction. Let $k$ be a totally real number field. Let $p$ be a fixed prime number and $\mathbb{Z}_{p}$ the ring of all $p$-adic integers. We denote by $\lambda=\lambda_{p}(k)$, $\mu=\mu_{p}(k)$ and $\nu=\nu_{p}(k)$ the Iwasawa invariants of the cyclotomic $\mathbb{Z}_{p^{-}}$ extension $k_{\infty}$ of $k$ for $p$ (cf. [10]).

Then Greenberg's conjecture states that both $\lambda_{p}(k)$ and $\mu_{p}(k)$ always vanish (cf. [8]). In other words, the order of the $p$-primary part of the ideal class group of $k_{n}$ remains bounded as $n$ tends to infinity, where $k_{n}$ is the $n$th layer of $k_{\infty} / k$. We know by the Ferrero-Washington theorem (cf. [2], [15]) that $\mu_{p}(k)$ always vanishes when $k$ is an abelian (not necessarily totally real) number field. However, the conjecture remains unsolved up to now except for some special cases (cf. [1], [3], [5]-[8], [13]).

This paper is a continuation of our previous papers [3], [5]-[7] and [12], that is to say, we investigate Greenberg's conjecture when $k$ is a real quadratic field and $p$ is an odd prime number which splits in $k$. The purpose of this paper is to extend our previous results, and to give basic numerical data of $k=\mathbb{Q}(\sqrt{m})$ for $0 \leq m \leq 10000$ and $p=3$. On the basis of these data, we can verify Greenberg's conjecture for most of these $k$ 's.

2. Notation and statement of the results. Let $k$ be a real quadratic field with class number $h$ and $\varepsilon$ the fundamental unit of $k$. Let $p$ be an odd prime number which splits in $k$, namely, $(p)=\mathfrak{p p}^{\prime}$ in $k$ where $\mathfrak{p} \neq \mathfrak{p}^{\prime}$. Then we can choose $\alpha \in k$ such that $\mathfrak{p}^{h}=(\alpha)$. In [6], we defined two invariants $n_{1}, n_{2} \in \mathbb{N}$ for $k$ and $p$ by

$$
\mathfrak{p}^{n_{1}}\left\|\left(\alpha^{p-1}-1\right), \quad \mathfrak{p}^{n_{2}}\right\|\left(\varepsilon^{p-1}-1\right) .
$$

Here $\mathfrak{p}^{n} \| \mathfrak{a}$ means that $\mathfrak{p}^{n} \mid \mathfrak{a}$ and $\mathfrak{p}^{n+1} \nmid \mathfrak{a}$ for an ideal $\mathfrak{a}$ of $k$. In spite of ambiguity of $\alpha, n_{1}$ is uniquely determined under the condition $n_{1} \leq n_{2}$.

1991 Mathematics Subject Classification: 11R23, 11R11, 11R27, 11Y40. 
For the cyclotomic $\mathbb{Z}_{p}$-extension

$$
k=k_{0} \subset k_{1} \subset \ldots \subset k_{n} \subset \ldots \subset k_{\infty}=\bigcup_{n=1}^{\infty} k_{n}
$$

with Galois group $\Gamma=\operatorname{Gal}\left(k_{\infty} / k\right)$, let $A_{n}$ be the $p$-primary part of the ideal class group of $k_{n}$, and $\mathfrak{p}_{n}$ (resp. $\mathfrak{p}_{n}^{\prime}$ ) the unique prime ideal of $k_{n}$ lying above $\mathfrak{p}\left(\right.$ resp. $\left.\mathfrak{p}^{\prime}\right)$. We put

$$
A_{n}^{\Gamma}=\left\{a \in A_{n} \mid a^{\sigma}=a \text { for all } \sigma \in \Gamma\right\} \quad \text { and } \quad D_{n}=\left\langle C l\left(\mathfrak{p}_{n}\right)\right\rangle \cap A_{n},
$$

where $C l\left(\mathfrak{p}_{n}\right)$ denotes the ideal class represented by $\mathfrak{p}_{n}$. Then we have $A_{n}^{\Gamma} \supset$ $D_{n}$. These groups are closely related to Greenberg's conjecture (cf. Theorem 2 in $[8])$.

Moreover, we introduce two other invariants $n_{0}^{(r)}$ and $n_{2}^{(r)}$ following [13]. Let $E_{n}$ be the group of units in $k_{n}$ and $d_{n}$ the order of $C l\left(\mathfrak{p}_{n}\right)$ (so the order of $\left.C l\left(\mathfrak{p}_{n}^{\prime}\right)\right)$ in the ideal class group of $k_{n}$. For each $m \geq n \geq 0$, we denote by $N_{m, n}$ the norm map from $k_{m}$ to $k_{n}$. Fix an integer $r \geq 0$. Then we can choose $\beta_{r} \in k_{r}$ such that $\mathfrak{p}_{r}^{\prime d_{r}}=\left(\beta_{r}\right)$. We define the invariants $n_{0}^{(r)}, n_{2}^{(r)} \in \mathbb{N}$ for $k$ and $p$ by

$$
\mathfrak{p}^{n_{0}^{(r)}} \|\left(N_{r, 0}\left(\beta_{r}\right)^{p-1}-1\right), \quad p^{n_{2}^{(r)}}=p^{n_{2}}\left(E_{0}: N_{r, 0}\left(E_{r}\right)\right) .
$$

As in the case of $n_{1}, n_{0}^{(r)}$ is uniquely determined under the condition $n_{0}^{(r)} \leq$ $n_{2}^{(r)}$, though the choice of $\beta_{r}$ is not unique. Here we note that $r+1 \leq n_{0}^{(r)}$ because $k_{\infty} / k$ is totally ramified at $p$. Furthermore, it is easy to see that

$$
n_{0}^{(r)} \leq n_{0}^{(r+1)} \leq n_{0}^{(r)}+1 \quad \text { and } \quad n_{2}^{(r)} \leq n_{2}^{(r+1)} \leq n_{2}^{(r)}+1
$$

for each $r \geq 0$. Put $n_{0}=n_{0}^{(0)}$ in particular. We then see that $n_{0} \leq n_{1} \leq n_{2}$.

Remark 1. By the definitions of $n_{0}^{(r)}$ and $n_{2}^{(r)}$, we see that $n_{0}^{(r)}$ is the maximal integer $n$ such that $\mathfrak{p}^{n} \mid\left(N_{r, 0}\left(\beta_{r}\right)^{p-1}-1\right)$ for all elements $\beta_{r}$ of $k_{r}$ satisfying $\mathfrak{p}_{r}^{\prime d_{r}}=\left(\beta_{r}\right)$ and that $n_{2}^{(r)}$ is the maximal integer $n$ such that $\mathfrak{p}^{n} \mid\left(N_{r, 0}\left(\varepsilon_{r}\right)^{p-1}-1\right)$ for all elements $\varepsilon_{r}$ of $E_{r}$. Indeed, it follows from the definition of $n_{2}^{(r)}$ that $\mathfrak{p}^{n_{2}^{(r)}} \mid\left(N_{r, 0}\left(\varepsilon_{r}\right)^{p-1}-1\right)$ for all $\varepsilon_{r} \in E_{r}$. Moreover, there exists $\eta_{r} \in E_{r}$ such that $\varepsilon^{u_{r}}=N_{r, 0}\left(\eta_{r}\right)$, so that $\mathfrak{p}^{n_{2}^{(r)}} \|\left(N_{r, 0}\left(\eta_{r}\right)^{p-1}-1\right)$, where $u_{r}$ denotes the integer such that $p^{u_{r}}=\left(E_{0}: N_{r, 0}\left(E_{r}\right)\right)$. Hence the second assertion follows. The first one immediately follows from the inequality $n_{0}^{(r)} \leq n_{2}^{(r)}$.

Remark 2. When we put $r=0$, we have

$$
n_{1}=\min \left\{n_{0}+v_{p}(h)-v_{p}\left(d_{0}\right), n_{2}\right\}
$$

where $v_{p}(a)$ denotes the exact power of $p$ dividing $a$. Hence, if $A_{0}=D_{0}$, then $n_{0}=n_{1}$. 
Let $\zeta_{p}$ be a primitive $p$ th root of unity and $k^{*}=k\left(\zeta_{p}\right)$. For the $C M$ field $k^{*}$, let $\left(k^{*}\right)^{+}$be the maximal real subfield of $k^{*}$ and put $\lambda_{p}^{-}\left(k^{*}\right)=$ $\lambda_{p}\left(k^{*}\right)-\lambda_{p}\left(\left(k^{*}\right)^{+}\right)$. Our main theorems are as follows.

Theorem 1 (Generalization of Proposition in [3] and Theorem 2 in [12]). Let $k$ be a real quadratic field and $p$ an odd prime number which splits in $k$. Assume that

(i) $\lambda_{p}^{-}\left(k^{*}\right)=1$ and

(ii) $n_{0}^{(r)} \neq n_{2}^{(r)}$ for some $r \geq 0$.

Then $\lambda_{p}(k)=\mu_{p}(k)=0$.

Remark 3. Let $\chi$ be the non-trivial Dirichlet character associated with $k$ and $\omega$ the Teichmüller character of $\operatorname{Gal}\left(\mathbb{Q}\left(\zeta_{p}\right) / \mathbb{Q}\right)$. We denote by $\lambda_{p}\left(k^{*}\right)_{\omega \chi^{-1}}$ the $\omega \chi^{-1}$-component of $\lambda_{p}\left(k^{*}\right)$. Then we may replace assumption (i) of Theorem 1 by a weaker assumption that $\lambda_{p}\left(k^{*}\right)_{\omega \chi^{-1}}=1$ (cf. Proposition 1 in $[9])$.

Putting $r=0$ in Theorem 1, we obtain the following

Corollary 1 (cf. Theorem 2 in [6]). Let $k$ and $p$ be as in Theorem 1. If $\lambda_{p}^{-}\left(k^{*}\right)=1$ and $n_{0} \neq n_{2}$, then $\lambda_{p}(k)=\mu_{p}(k)=0$.

THEOREM 2. Let $k$ be a real quadratic field and $p$ an odd prime number which splits in $k$. Assume that $A_{0}=D_{0}$. Then the following conditions are equivalent.

(i) $n_{0}^{(r)}=r+1$ for some $r \geq 0$.

(ii) $n_{0}^{(r)}=r+1$ for all sufficiently large $r$.

(iii) $n_{2}^{(r)}=r+1$ for some $r \geq 0$.

(iv) $n_{2}^{(r)}=r+1$ for all sufficiently large $r$.

(v) $A_{n}^{\Gamma}=D_{n}$ for all sufficiently large $n$.

In particular, one of these conditions holds if and only if $\lambda_{p}(k)=\mu_{p}(k)=0$.

Putting $r=0$ in the condition (i) of Theorem 2, we obtain the following.

Corollary 2 (cf. Theorem 1 in [6]). Let $k$ and $p$ be as in Theorem 2. If $A_{0}=D_{0}$ and $n_{0}=1$ (i.e., $n_{1}=1$ ), then $\lambda_{p}(k)=\mu_{p}(k)=0$.

Moreover, putting $r=n_{2}-1$ in condition (iii) of Theorem 2, we obtain the following by Lemma 8 (cf. Section 5 ).

Corollary 3 (cf. Theorem in [5] and Lemma in [7]). Let $k$ and $p$ be as in Theorem 2. If $A_{0}=D_{0}$ and $N_{n_{2}-1,0}\left(E_{n_{2}-1}\right)=E_{0}$, then $\lambda_{p}(k)=\mu_{p}(k)=0$.

The notation defined in this section will be used throughout this paper. We also denote by $\beta_{r} \in k_{r}$ a generator of $\mathfrak{p}_{r}^{\prime d_{r}}$ satisfying

$$
\mathfrak{p}^{n_{0}^{(r)}} \|\left(N_{r, 0}\left(\beta_{r}\right)^{p-1}-1\right) \quad \text { and } \quad n_{0}^{(r)} \leq n_{2}^{(r)} .
$$


Namely, $\beta_{r} \in k_{r}$ is a generator of $\mathfrak{p}_{r}^{\prime d_{r}}$ which determines $n_{0}^{(r)}$. Since $p$ splits in $k$, we have $k_{\mathfrak{p}} \simeq \mathbb{Q}_{p}$, where $k_{\mathfrak{p}}$ is the completion of $k$ at $\mathfrak{p}$. So, by identifying $\mathfrak{p} \in k_{\mathfrak{p}}$ with $p \in \mathbb{Q}_{p}$, we may write $N_{r, 0}\left(\beta_{r}\right)^{p-1} \in k$ as in the following form of a $p$-adic integer:

$$
N_{r, 0}\left(\beta_{r}\right)^{p-1}=1+p^{n_{0}^{(r)}} x_{r}, \quad x_{r} \in \mathbb{Z}_{p}^{\times} .
$$

3. Some fundamental lemmas. We first refer to the following three lemmas.

Lemma 1 (cf. Theorem 2 in [8]). Let $k$ and $p$ be as in Section 2. Then $A_{n}^{\Gamma}=D_{n}$ for all sufficiently large $n$ if and only if $\lambda_{p}(k)=\mu_{p}(k)=0$.

Lemma 2 (cf. Proposition 1 in [6]). Let $k$ and $p$ be as in Section 2. Then

$$
\left|A_{n}^{\Gamma}\right|= \begin{cases}\left|A_{0}\right| p^{n} & \text { if } n<n_{2}-1, \\ \left|A_{0}\right| p^{n_{2}-1} & \text { if } n \geq n_{2}-1 .\end{cases}
$$

Lemma 3 (cf. Lemma 3 in [12]). Let $k$ and $p$ be as in Section 2. If $A_{n}$ is cyclic for all $n \geq 0$ and if $D_{r}$ is non-trivial for some $r \geq 0$, then $\lambda_{p}(k)=\mu_{p}(k)=0$.

Next we prove two more lemmas. Since $\mathfrak{p}_{r}=\mathfrak{p}_{r+1}^{p}$, we have $d_{r+1}=d_{r}$ or $p d_{r}$; in particular, $\left|D_{r+1}\right|=\left|D_{r}\right|$ or $p\left|D_{r}\right|$. If we write $d_{r}=c p^{j}$ with an integer $c$ prime to $p$, then $c$ is independent of $r$.

Lemma 4. Let $r$ be a fixed non-negative integer. Assume that $\left|D_{r+1}\right|=$ $p\left|D_{r}\right|$. Then

$$
n_{0}^{(r+1)}= \begin{cases}n_{0}^{(r)} & \text { if } n_{0}^{(r)}=n_{2}^{(r)}=n_{2}^{(r+1)}, \\ n_{0}^{(r)}+1 & \text { otherwise. }\end{cases}
$$

Pr o of. Since $d_{r+1}=p d_{r}$, we have

$$
\mathfrak{p}_{r+1}^{\prime d_{r+1}}=\mathfrak{p}_{r+1}^{\prime p d_{r}}=\mathfrak{p}_{r}^{\prime d_{r}}=\left(\beta_{r}\right) \quad \text { in } k_{r+1} .
$$

Thus we may take $\beta_{r}$ as a generator of $\mathfrak{p}_{r+1}^{\prime d_{r+1}}$. Then we obtain

$$
\begin{aligned}
& N_{r+1,0}\left(\beta_{r}\right)^{p-1}=N_{r, 0}\left(\beta_{r}\right)^{p(p-1)} \\
& =\left(1+p^{n_{0}^{(r)}} x_{r}\right)^{p}, \quad x_{r} \in \mathbb{Z}_{p}^{\times}, \\
& =1+p^{n_{0}^{(r)}+1} x_{r}^{\prime}, \quad x_{r}^{\prime} \in \mathbb{Z}_{p}^{\times},
\end{aligned}
$$

therefore

$$
\mathfrak{p}^{n_{0}^{(r)}+1} \|\left(N_{r+1,0}\left(\beta_{r}\right)^{p-1}-1\right) .
$$

Hence it follows from the definition of $n_{0}^{(r+1)}$ that

$$
n_{0}^{(r+1)}=\min \left\{n_{0}^{(r)}+1, n_{2}^{(r+1)}\right\},
$$

which yields the desired result. 
Lemma 5. Let $r$ be a fixed non-negative integer. Assume that $\left|D_{r}\right|=$ $\left|D_{r+1}\right|$. Then

(i) If $n_{0}^{(r)}<n_{2}^{(r)}$, then $n_{0}^{(r+1)}=n_{0}^{(r)}$.

(ii) If $n_{2}^{(r)}=n_{2}^{(r+1)}$, then $n_{0}^{(r+1)}=n_{0}^{(r)}$.

Proof. (i) Since $d_{r+1}=d_{r}$, we have

$$
\left(\beta_{r}\right)=\mathfrak{p}_{r}^{\prime d_{r}}=N_{r+1, r}\left(\mathfrak{p}_{r+1}^{\prime d_{r}}\right)=N_{r+1, r}\left(\mathfrak{p}_{r+1}^{\prime d_{r+1}}\right)=\left(N_{r+1, r}\left(\beta_{r+1}\right)\right) \quad \text { in } k_{r} .
$$

Hence $N_{r+1, r}\left(\beta_{r+1}\right)=\beta_{r} \varepsilon_{r}$ for some $\varepsilon_{r} \in E_{r}$. Taking the norm from $k_{r}$ to $k$, we have $N_{r+1,0}\left(\beta_{r+1}\right)=N_{r, 0}\left(\beta_{r}\right) N_{r, 0}\left(\varepsilon_{r}\right)$. Therefore we obtain the following $p$-adic expansion:

(1) $1+p^{n_{0}^{(r+1)}} x_{r+1}=1+p^{n_{0}^{(r)}} x_{r}+p^{n_{2}^{(r)}} y_{r}+\ldots, \quad x_{r}, x_{r+1} \in \mathbb{Z}_{p}^{\times}, y_{r} \in \mathbb{Z}_{p}$.

This implies the desired result.

(ii) Suppose that $n_{0}^{(r+1)} \neq n_{0}^{(r)}$. Then it follows from (1) that $n_{0}^{(r)}=n_{2}^{(r)}$. Therefore $n_{0}^{(r+1)}>n_{0}^{(r)}=n_{2}^{(r)}=n_{2}^{(r+1)}$, which contradicts the definition of $n_{0}^{(r+1)}$. This completes the proof.

Remark 4 . Lemmas 4 and 5 can be used for determining $n_{0}^{(r+1)}$ from $n_{0}^{(r)}, n_{2}^{(r)}$ and $n_{2}^{(r+1)}$. However, Lemma 5 does not work in the case where $n_{0}^{(r)}=n_{2}^{(r)}<n_{2}^{(r+1)}$. Actually, when $p=3$, we see that $n_{0}=n_{2}=2<$ $n_{2}^{(1)}=3$ and $n_{0}^{(1)}=2$ for $k=\mathbb{Q}(\sqrt{106})$, and that $n_{0}=n_{2}=2<n_{2}^{(1)}=3$ and $n_{0}^{(1)}=3$ for $k=\mathbb{Q}(\sqrt{295})$ (cf. Table 1$)$. Hence, in this situation the practical calculation of $\beta_{r+1}$ is necessary to the determination of $n_{0}^{(r+1)}$.

4. The proof of Theorem 1 and some examples. In order to prove Theorem 1, we need the following lemma.

LEMMA 6. Let $r$ be a fixed non-negative integer. If $n_{0}^{(r)} \neq n_{2}^{(r)}$, then $\left|D_{n}\right|>\left|D_{r}\right|$ for all $n \geq n_{0}^{(r)}$.

Proof. Suppose that $\left|D_{n}\right|=\left|D_{r}\right|$ for some $n \geq n_{0}^{(r)}$. Then since $d_{n}=d_{r}$, we have $N_{n, r}\left(\beta_{n}\right)=\beta_{r} \varepsilon_{r}$ for some $\varepsilon_{r} \in E_{r}$, as in the proof of Lemma 5 . Taking the norm and expanding it in the $p$-adic form, we obtain

(2) $1+p^{n_{0}^{(n)}} x_{n}=1+p^{n_{0}^{(r)}} x_{r}+p^{n_{2}^{(r)}} y_{r}+\ldots, \quad x_{r}, x_{n} \in \mathbb{Z}_{p}^{\times}, y_{r} \in \mathbb{Z}_{p}$.

Since $n_{0}^{(n)} \geq n+1 \geq n_{0}^{(r)}+1>n_{0}^{(r)}$ for all $n \geq n_{0}^{(r)}$, it follows from (2) that $n_{0}^{(r)}=n_{2}^{(r)}$. This completes the proof.

By Lemma $2,\left|A_{n}^{\Gamma}\right|$ remains bounded as $n$ tends to infinity, hence so does $\left|D_{n}\right|$. Therefore we obtain the following as a corollary to Lemma 6 . 
COROLlary 4. Let $k$ and $p$ be as in Section 2. Then $n_{0}^{(r)}=n_{2}^{(r)}$ for all sufficiently large $r$.

Proof of Theorem 1 . Let $k_{n}^{*}$ be the $n$th layer of the cyclotomic $\mathbb{Z}_{p^{-}}$-extension $k_{\infty}^{*} / k^{*}$ and $A_{n}^{*}$ the $p$-primary part of the ideal class group of $k_{n}^{*}$. Since $k_{n}^{*}$ is a $C M$-field, we can define $\left(A_{n}^{*}\right)^{+}$by the $p$-primary part of the ideal class group of its maximal real subfield and $\left(A_{n}^{*}\right)^{-}$by the kernel of the norm map from $A_{n}^{*}$ to $\left(A_{n}^{*}\right)^{+}$. The Ferrero-Washington theorem guarantees the vanishing of $\mu_{p}\left(k^{*}\right)$, hence, by assumption (i), $\left(A_{n}^{*}\right)^{-}$is cyclic for all $n \geq 0$. It follows from the reflection theorem that $\left(A_{n}^{*}\right)^{+}$is cyclic, hence so is $A_{n}$ for all $n \geq 0$. By Lemma 6 , we also have the inequality $\left|D_{n}\right|>\left|D_{r}\right|$ under assumption (ii), hence $D_{n} \neq 1$, for all $n \geq n_{0}^{(r)}$. Therefore Theorem 1 immediately follows from Lemma 3.

EXAMPLE 1 . Let $k=\mathbb{Q}(\sqrt{26893})$ and $p=3$, for which we could not verify that $\lambda_{3}(k)=\mu_{3}(k)=0$ in [13]. Then $n_{0}=n_{1}=n_{2}=4$, and moreover, $\lambda_{3}^{-}\left(k^{*}\right)=1$ and $n_{0}^{(1)}=4 \neq n_{2}^{(1)}=5$ (see Table 2 of [13]). Therefore it follows from Theorem 1 that $\lambda_{3}(k)=\mu_{3}(k)=0$.

ExAmple 2. Let $k=\mathbb{Q}(\sqrt{4651})$ and $p=3$. Then $\lambda_{3}^{-}\left(k^{*}\right)=1$ and $n_{0}=1 \neq n_{1}=n_{2}=2$ (see Table 1$)$. Therefore it follows from Theorem 1 that $\lambda_{3}(k)=\mu_{3}(k)=0$. Note that $\left|A_{0}\right|=3>1=\left|D_{0}\right|$. In order to conclude that $\lambda_{3}(k)=\mu_{3}(k)=0$ for this $k$, we needed the information on the initial layer $k_{1}$ of $k_{\infty} / k$ before now (cf. [3], [7]). But we do not need such information now, therefore it seems that the invariant $n_{0}$ is more useful than $n_{1}$.

5. The proof of Theorem 2. First, we prove the following lemma.

Lemma 7. Let $r$ and $s$ be fixed non-negative integers. If $\left|D_{r+s}\right|=p^{t}\left|D_{r}\right|$, then

$$
n_{0}^{(r)} \geq \min \left\{n_{0}^{(r+s)}-t, n_{2}^{(r)}-t\right\} .
$$

Proof. Note that $s \geq t$ and $d_{r+s}=p^{t} d_{r}$. Then we have

$$
\left(\beta_{r+s}^{p^{s-t}}\right)=\mathfrak{p}_{r+s}^{\prime p^{s-t}} d_{r+s}=\mathfrak{p}_{r+s}^{\prime p^{s} d_{r}}=\mathfrak{p}_{r}^{\prime d_{r}}=\left(\beta_{r}\right) \quad \text { in } k_{r+s},
$$

hence $\left(N_{r+s, r}\left(\beta_{r+s}\right)\right)^{p^{s-t}}=\left(\beta_{r}\right)^{p^{s}}$. So $\left(N_{r+s, r}\left(\beta_{r+s}\right)\right)=\left(\beta_{r}\right)^{p^{t}}$ in $k_{r}$. Therefore

$$
\beta_{r}^{p^{t}}=N_{r+s, r}\left(\beta_{r+s}\right) \varepsilon_{r} \quad \text { for some } \varepsilon_{r} \in E_{r} .
$$

Taking the norm and expanding it in the $p$-adic form, we obtain

$$
1+p^{n_{0}^{(r)}+t} x_{r}^{\prime}=1+p^{n_{0}^{(r+s)}} x_{r+s}+p^{n_{2}^{(r)}} y_{r}+\ldots, \quad x_{r}^{\prime}, x_{r+s} \in \mathbb{Z}_{p}^{\times}, y_{r} \in \mathbb{Z}_{p} .
$$

This immediately implies Lemma 7. 
From now on, we consider the case where $A_{0}=D_{0}$. Let $\bar{A}_{n}^{\Gamma}$ be the subgroup of $A_{n}$ consisting of ideal classes which contain an ideal invariant under the action of $\operatorname{Gal}\left(k_{n} / k\right)$. Then the genus formula (cf. [16]) says that

$$
\left|\bar{A}_{n}^{\Gamma}\right|=\left|A_{0}\right| \frac{p^{n}}{\left(E_{0}: N_{n, 0}\left(E_{n}\right)\right)} .
$$

If $A_{0}=D_{0}$, then $\bar{A}_{n}^{\Gamma}=D_{n}$ for all $n \geq 0$ because $\bar{A}_{n}^{\Gamma}=i_{0, n}\left(A_{0}\right) D_{n}$, where $i_{0, n}$ denotes the natural map from the ideal group of $k$ to the ideal group of $k_{n}$ induced from the inclusion map. Hence we immediately obtain the following lemmas.

Lemma 8. Let $r$ be a fixed non-negative integer. Assume that $A_{0}=D_{0}$. Then

(i) $\left|D_{r}\right|=\left|D_{0}\right| p^{r-u_{r}}=\left|D_{0}\right| p^{n_{2}+r-n_{2}^{(r)}}$,

(ii) $n_{2}^{(r)}=n_{2}+r-u$,

where $u_{r}$ is the integer such that $p^{u_{r}}=\left(E_{0}: N_{r, 0}\left(E_{r}\right)\right)$ and $u$ is the integer such that $\left|D_{r}\right|=p^{u}\left|D_{0}\right|$.

Lemma 9. Let $r$ be a fixed non-negative integer. Assume that $A_{0}=D_{0}$. Then $\left|D_{r+1}\right|=p\left|D_{r}\right|$ if and only if $n_{2}^{(r+1)}=n_{2}^{(r)}$.

Proof. Let $u_{r}$ be as in Lemma 8. Then $\left|D_{r+1}\right|=p\left|D_{r}\right|$ if and only if $u_{r+1}=u_{r}$. Hence the result follows from the definition of $n_{2}^{(r)}$.

Lemma 10. Let $r$ be a fixed non-negative integer. Assume that $A_{0}=D_{0}$ and that $\left|D_{r+1}\right|=p\left|D_{r}\right|$. Then $n_{0}^{(r+1)}=n_{0}^{(r)}$ if and only if $n_{0}^{(r)}=n_{2}^{(r)}$. Namely, we have

$$
n_{0}^{(r+1)}= \begin{cases}n_{0}^{(r)} & \text { if } n_{0}^{(r)}=n_{2}^{(r)}, \\ n_{0}^{(r)}+1 & \text { if } n_{0}^{(r)} \neq n_{2}^{(r)} .\end{cases}
$$

Pr o of. This easily follows from Lemmas 4 and 9.

Proof of Theorem 2 . (iv) $\Rightarrow$ (iii) $\Rightarrow$ (i) and (iv) $\Rightarrow($ ii) $\Rightarrow$ (i) are trivial. Therefore it is sufficient to prove that $(\mathrm{i}) \Rightarrow(\mathrm{v}) \Rightarrow(\mathrm{iv})$.

$(\mathrm{i}) \Rightarrow(\mathrm{v})$. Let $r$ be a non-negative integer such that $n_{0}^{(r)}=r+1$. Then $n_{0}^{(r+1)}=(r+1)+1$ because $(r+1)+1 \leq n_{0}^{(r+1)}$ and $n_{0}^{(r+1)} \leq n_{0}^{(r)}+1$. Repeating this process, we conclude that $n_{0}^{(r+s)}=r+s+1$ for all $s \geq 0$. We denote by $u$ the integer such that $\left|D_{r}\right|=p^{u}\left|D_{0}\right|$. For $s \geq n_{2}-1-u$, we put

$$
\left|D_{r+s}\right|=p^{t}\left|D_{r}\right|=p^{t+u}\left|D_{0}\right|
$$


Now suppose that $t+u<n_{2}-1$. Then we have

$$
\begin{aligned}
n_{0}^{(r+s)}-t & =r+s+1-t \geq r+n_{2}-u-t \geq r+2, \\
n_{2}^{(r)}-t & =n_{2}+r-u-t \geq r+2
\end{aligned}
$$

by Lemma 8(ii). It easily follows from Lemma 7 that

$$
n_{0}^{(r)} \geq \min \left\{n_{0}^{(r+s)}-t, n_{2}^{(r)}-t\right\} \geq r+2,
$$

which is a contradiction. Hence we must have $t+u=n_{2}-1$, so $\left|D_{r+s}\right|=$ $\left|D_{0}\right| p^{n_{2}-1}$ for all $s \geq n_{2}-1-u$. Therefore Lemma 2 implies that $A_{n}^{\Gamma}=D_{n}$ for all $n \geq n_{2}^{(r)}-1$.

$(\mathrm{v}) \Rightarrow$ (iv). By Lemma 2, we have

$$
\left|D_{r}\right|=\left|A_{r}^{\Gamma}\right|=\left|A_{0}\right| p^{n_{2}-1}=\left|D_{0}\right| p^{n_{2}-1}
$$

for all sufficiently large $r$. Hence Lemma 8(i) shows that

$$
\left|D_{0}\right| p^{n_{2}+r-n_{2}^{(r)}}=\left|D_{0}\right| p^{n_{2}-1}
$$

which means that $n_{2}^{(r)}=r+1$ for all sufficiently large $r$.

The last assertion immediately follows from Lemma 1. This completes the proof of Theorem 2 .

6. Other useful results and some examples. In this section we shall give a few of easy results, which are useful when we cannot apply Theorems 1 and 2. First we prove the following.

LEMmA 11. If there exists an integer $r_{0}$ such that $\left|A_{r_{0}}^{\Gamma}\right|=\left|D_{r_{0}}\right|$ and $r_{0} \geq n_{2}-1$, then $A_{n} \simeq A_{r_{0}}$ for all $n \geq r_{0}$.

Proof. Note that $N_{m, n}: A_{m} \rightarrow A_{n}$ and $N_{m, n}: D_{m} \rightarrow D_{n}$ are surjective for all $m \geq n \geq 0$ because $k_{\infty} / k$ is totally ramified at $p$. It follows from the assumption and Lemma 2 that $N_{m, n}: A_{m}^{\Gamma} \rightarrow A_{n}^{\Gamma}$ is isomorphic for all $m \geq n \geq r_{0}$. Hence, $N_{m, n}: A_{m} \rightarrow A_{n}$ is also isomorphic for all $m \geq n \geq r_{0}$. This completes the proof.

Proposition 1. Let $k$ and $p$ be as in Section 2. If $\left|D_{r}\right|=\left|A_{0}\right| p^{n_{2}-2}$ and $n_{0}^{(r)} \neq n_{2}^{(r)}$ for some $r \geq 0$, then $A_{n} \simeq A_{n_{0}^{(r)}}$ for all $n \geq n_{0}^{(r)}$, hence in particular $\lambda_{p}(k)=\mu_{p}(k)=0$.

Proof. It follows from Lemma 6 that $\left|D_{n}\right|>\left|D_{r}\right|=\left|A_{0}\right| p^{n_{2}-2}$ for all $n \geq n_{0}^{(r)}$. Hence $\left|A_{n}^{\Gamma}\right|=\left|A_{0}\right| p^{n_{2}-1}=\left|D_{n}\right|$ for all $n \geq n_{0}^{(r)}$ by Lemma 2 . Since $n_{0}^{(r)} \geq n_{2}-1$, the assertion immediately follows from Lemma 11 .

EXAMPLE 3. Let $k=\mathbb{Q}(\sqrt{7753})$ and $p=3$. Then $n_{0}=1 \neq n_{1}=n_{2}=2$, $\lambda_{3}^{-}\left(k^{*}\right)=2$ and $\left|A_{0}\right|=3>1=\left|D_{0}\right|$. Hence Theorems 1 and 2 cannot be applied to this $k$. However, $\left|D_{1}\right|=3=\left|A_{0}\right|$ and $n_{0}^{(1)}=2 \neq n_{2}^{(1)}=3$ (see 
Table 1). Therefore it follows from Proposition 1 that $A_{n} \simeq A_{2}$ for all $n \geq 2$, in particular $\lambda_{3}(k)=\mu_{3}(k)=0$.

Lemma 6 asserts that $n_{0}^{(r)} \neq n_{2}^{(r)}$ implies $\left|D_{r}\right|<\left|D_{n_{0}^{(r)}}\right|$. However, the converse does not always hold (cf. Example 4). Thus the following proposition is sometimes useful. Here we note that, if $A_{n}$ is cyclic for all $n \geq 0$ and if $A_{0}$ is trivial, then the converse is also true. In fact, for a fixed non-negative integer $r$, we see that $n_{0}^{(r)}=r+s$ if and only if $\left|D_{r}\right|=\ldots=\left|D_{r+s-1}\right|<\left|D_{r+s}\right|$ for $1 \leq s \leq n_{2}^{(r)}-r-1$ in this situation (cf. Theorem 1 of [12]).

Proposition 2. Let $k$ and $p$ be as in Section 2. If $\lambda_{p}^{-}\left(k^{*}\right)=1$, and $D_{r} \neq 1$ for some $r \geq 0$, then $\lambda_{p}(k)=\mu_{p}(k)=0$.

Proof. This immediately follows from the proof of Theorem 1 (or Lemma 3).

EXAmPle 4. Let $k=\mathbb{Q}(\sqrt{1129})$ and $p=3$. Then $n_{0}=n_{1}=n_{2}=1$, $n_{0}^{(1)}=n_{2}^{(1)}=2$ and $\left|A_{0}\right|=9>3=\left|D_{0}\right|$ (see Table 1 ). Hence Theorem 1 for $r=0,1$ and Theorem 2 cannot be applied to this $k$. But, since $\lambda_{3}^{-}\left(k^{*}\right)=1$, it follows from Proposition 2 that $\lambda_{3}(k)=\mu_{3}(k)=0$. Now, by Table 1 and Lemma 2, we see that $\left|A_{1}^{\Gamma}\right|=9=\left|D_{1}\right|$, so $\left|A_{n}^{\Gamma}\right|=\left|D_{n}\right|=\left|D_{1}\right|$ for all $n \geq 1$. Therefore Lemma 6 implies that $n_{0}^{(r)}=n_{2}^{(r)}=r+1$ for all $r \geq 1$, so all $r \geq 0$. Hence we cannot apply Theorem 1 for all $r \geq 0$ to this $k$.

Finally we note that there exist some examples of $k$ to which we cannot apply our theorems and propositions, but nevertheless we can verify Greenberg's conjecture for them by Lemma 11 . Such examples are $k=\mathbb{Q}(\sqrt{6601})$, $k=\mathbb{Q}(\sqrt{6901})$ and so on.

7. Tables of basic numerical data of $k=\mathbb{Q}(\sqrt{m})$ for $p=3$. We shall give a table of the fundamental data of $k=\mathbb{Q}(\sqrt{m})$ for $p=3$ and positive square-free integers $m$ 's less than 10000 satisfying $m \equiv 1(\bmod 3)$. The total number of such $m$ 's is exactly 2279. We find that there exist exactly 2042 $m$ 's which satisfy $A_{0}=D_{0}$ and $n_{0}=1$. Greenberg's conjecture is valid for these $k$ 's by Corollary 2 to Theorem 2. Table 1 gives several useful data for 237 remaining $m$ 's. We can verify Greenberg's conjecture for $185 k$ 's in Table 1 by applying our results. The asterisks in the column of $\lambda_{3}(k)$, the number of which is exactly 52 , mean that Greenberg's conjecture cannot be verified by these data.

Concerning our method of computation, we refer to [11] and [13] for $n_{0}^{(1)}, n_{2}^{(1)},\left|A_{1}\right|$ and $\left|D_{1}\right|$, to [14] for the 3-primary part $A_{0}^{*-}$ of the ideal class group of $\mathbb{Q}(\sqrt{-3 m})$, and to $[4]$ for $\lambda_{3}^{-}\left(k^{*}\right)$. Note that $\lambda_{3}^{-}\left(k^{*}\right)=$ $\lambda_{3}(\mathbb{Q}(\sqrt{-3 m}))$. The rest is easily computed. 
Addendum. Recently, after we have written this paper, we heard from H. Sumida that he verified Greenberg's conjecture for $p=3$ and $m=$ $727,2794,4279,4741,5533,7429,7465,7642,9634$ and 9691, which are marked with the asterisks in Table 1, by computing the Iwasawa polynomials associated with $p$-adic $L$-functions. He is now preparing the paper entitled "Greenberg's conjecture and the Iwasawa polynomial".

Table 1. All $m$ 's satisfying $A_{0} \neq D_{0}$ or $n_{0}>1: 1 \leq m \leq 10000$

\begin{tabular}{|c|c|c|c|c|c|c|c|c|c|c|c|c|}
\hline$m$ & $n_{0}$ & $n_{1}$ & $n_{2}$ & $n_{0}^{(1)}$ & $n_{2}^{(1)}$ & $\lambda_{3}^{-}\left(k^{*}\right)$ & $A_{0}^{*-}$ & $\left|D_{0}\right|$ & $\left|A_{0}\right|$ & $\left|D_{1}\right|$ & $\left|A_{1}\right|$ & $\lambda_{3}(k)$ \\
\hline 67 & 2 & 2 & 3 & 2 & 4 & 1 & (3) & 1 & 1 & 1 & 3 & 0 \\
\hline 103 & 2 & 2 & 2 & 2 & 2 & 2 & (3) & 1 & 1 & 3 & 9 & 0 \\
\hline 106 & 2 & 2 & 2 & 2 & 3 & 1 & (3) & 1 & 1 & 1 & 3 & 0 \\
\hline 139 & 2 & 2 & 2 & 2 & 2 & 2 & (3) & 1 & 1 & 3 & 9 & 0 \\
\hline 238 & 2 & 2 & 3 & 2 & 4 & 1 & (3) & 1 & 1 & 1 & 3 & 0 \\
\hline 253 & 2 & 2 & 2 & 2 & 3 & 1 & (3) & 1 & 1 & 1 & 3 & 0 \\
\hline 295 & 2 & 2 & 2 & 3 & 3 & 1 & (3) & 1 & 1 & 1 & 3 & * \\
\hline 397 & 2 & 2 & 2 & 3 & 3 & 1 & (3) & 1 & 1 & 1 & 3 & * \\
\hline 418 & 2 & 2 & 2 & 2 & 2 & 2 & (3) & 1 & 1 & 3 & 9 & 0 \\
\hline 454 & 2 & 2 & 2 & 2 & 3 & 1 & (3) & 1 & 1 & 1 & 3 & 0 \\
\hline 505 & 2 & 2 & 2 & 2 & 3 & 1 & (3) & 1 & 1 & 1 & 3 & 0 \\
\hline 607 & 2 & 2 & 2 & 2 & 3 & 1 & (9) & 1 & 1 & 1 & 3 & 0 \\
\hline 610 & 2 & 2 & 4 & 2 & 5 & 1 & (3) & 1 & 1 & 1 & 3 & 0 \\
\hline 679 & 2 & 2 & 2 & 2 & 2 & 2 & (3) & 1 & 1 & 3 & 9 & 0 \\
\hline 727 & 2 & 2 & 3 & 3 & 3 & 2 & (9) & 1 & 1 & 3 & 9 & * \\
\hline 745 & 2 & 2 & 2 & 3 & 3 & 1 & (3) & 1 & 1 & 1 & 3 & * \\
\hline 787 & 2 & 2 & 2 & 2 & 3 & 1 & (9) & 1 & 1 & 1 & 3 & 0 \\
\hline 790 & 2 & 2 & 2 & 2 & 2 & 2 & (3) & 1 & 1 & 3 & 9 & 0 \\
\hline 886 & 2 & 2 & 2 & 2 & 3 & 1 & (3) & 1 & 1 & 1 & 3 & 0 \\
\hline 994 & 2 & 2 & 2 & 2 & 3 & 1 & (3) & 1 & 1 & 1 & 3 & 0 \\
\hline 1102 & 2 & 2 & 2 & 2 & 3 & 1 & (3) & 1 & 1 & 1 & 3 & 0 \\
\hline 1129 & 1 & 1 & 1 & 2 & 2 & 1 & (3) & 3 & 9 & 9 & 27 & 0 \\
\hline 1153 & 2 & 2 & 2 & 2 & 2 & 2 & (3) & 1 & 1 & 3 & 9 & 0 \\
\hline 1261 & 2 & 2 & 2 & 2 & 2 & 2 & (3) & 1 & 1 & 3 & 9 & 0 \\
\hline 1294 & 2 & 2 & 2 & 2 & 3 & 1 & (3) & 1 & 1 & 1 & 3 & 0 \\
\hline 1318 & 2 & 2 & 2 & 2 & 3 & 1 & (3) & 1 & 1 & 1 & 3 & 0 \\
\hline 1333 & 2 & 2 & 2 & 2 & 3 & 1 & (3) & 1 & 1 & 1 & 3 & 0 \\
\hline 1390 & 3 & 3 & 4 & 3 & 5 & 1 & (3) & 1 & 1 & 1 & 3 & 0 \\
\hline 1462 & 2 & 2 & 2 & 2 & 3 & 1 & (3) & 1 & 1 & 1 & 3 & 0 \\
\hline 1609 & 2 & 2 & 2 & 2 & 2 & 4 & (3) & 1 & 1 & 3 & 9 & 0 \\
\hline 1642 & 2 & 2 & 2 & 2 & 2 & 2 & (3) & 1 & 1 & 3 & 27 & 0 \\
\hline 1654 & 1 & 1 & 1 & 2 & 2 & 1 & (3) & 3 & 9 & 9 & 27 & 0 \\
\hline 1669 & 2 & 2 & 2 & 2 & 3 & 1 & (9) & 1 & 1 & 1 & 3 & 0 \\
\hline 1714 & 2 & 2 & 2 & 3 & 3 & 4 & $(3,3)$ & 3 & 3 & 3 & 9 & $*$ \\
\hline 1726 & 2 & 2 & 2 & 2 & 2 & 2 & (3) & 1 & 1 & 3 & 27 & 0 \\
\hline 1738 & 2 & 2 & 2 & 3 & 3 & 1 & (9) & 1 & 1 & 1 & 3 & $*$ \\
\hline 1753 & 2 & 2 & 2 & 2 & 3 & 1 & (3) & 1 & 1 & 1 & 3 & 0 \\
\hline 1810 & 2 & 2 & 2 & 2 & 3 & 1 & (9) & 1 & 1 & 1 & 3 & 0 \\
\hline
\end{tabular}


Table 1 (cont.)

\begin{tabular}{|c|c|c|c|c|c|c|c|c|c|c|c|c|}
\hline$m$ & $n_{0}$ & $n_{1}$ & $n_{2}$ & $n_{0}^{(1)}$ & $n_{2}^{(1)}$ & $\lambda_{3}^{-}\left(k^{*}\right)$ & $A_{0}^{*-}$ & $\left|D_{0}\right|$ & $\left|A_{0}\right|$ & $\left|D_{1}\right|$ & $\left|A_{1}\right|$ & $\lambda_{3}(k)$ \\
\hline 1867 & 2 & 2 & 6 & 2 & 7 & 1 & $(3)$ & 1 & 1 & 1 & 3 & 0 \\
\hline 1894 & 2 & 2 & 3 & 2 & 4 & 1 & (3) & 1 & 1 & 1 & 3 & 0 \\
\hline 1954 & 1 & 1 & 1 & 2 & 2 & 1 & (3) & 1 & 3 & 3 & 9 & 0 \\
\hline 2029 & 2 & 2 & 2 & 3 & 3 & 1 & (9) & 1 & 1 & 1 & 3 & $*$ \\
\hline 2059 & 3 & 3 & 3 & 4 & 4 & 1 & (3) & 1 & 1 & 1 & 3 & * \\
\hline 2122 & 2 & 2 & 2 & 2 & 3 & 2 & (3) & 1 & 1 & 1 & 9 & 0 \\
\hline 2149 & 4 & 4 & 4 & 5 & 5 & 1 & (3) & 1 & 1 & 1 & 3 & $*$ \\
\hline 2158 & 2 & 2 & 2 & 2 & 3 & 1 & (3) & 1 & 1 & 1 & 3 & 0 \\
\hline 2221 & 2 & 2 & 3 & 2 & 4 & 1 & (3) & 1 & 1 & 1 & 3 & 0 \\
\hline 2230 & 2 & 2 & 2 & 2 & 3 & 2 & $(3,3)$ & 3 & 3 & 3 & 9 & 0 \\
\hline 2263 & 2 & 2 & 2 & 2 & 3 & 2 & $(3,3)$ & 3 & 3 & 3 & 9 & 0 \\
\hline 2371 & 2 & 2 & 2 & 2 & 3 & 1 & (9) & 1 & 1 & 1 & 3 & 0 \\
\hline 2410 & 2 & 2 & 3 & 2 & 4 & 1 & (3) & 1 & 1 & 1 & 3 & 0 \\
\hline 2419 & 1 & 1 & 1 & 2 & 2 & 1 & (9) & 1 & 3 & 3 & 9 & 0 \\
\hline 2431 & 2 & 2 & 2 & 2 & 2 & 3 & (3) & 1 & 1 & 3 & 9 & 0 \\
\hline 2515 & 2 & 2 & 2 & 2 & 3 & 1 & (9) & 1 & 1 & 1 & 3 & 0 \\
\hline 2521 & 2 & 2 & 3 & 2 & 4 & 1 & (3) & 1 & 1 & 1 & 3 & 0 \\
\hline 2593 & 2 & 2 & 3 & 2 & 4 & 1 & (3) & 1 & 1 & 1 & 3 & 0 \\
\hline 2659 & 2 & 2 & 3 & 2 & 4 & 2 & $(3,3)$ & 3 & 3 & 3 & 9 & 0 \\
\hline 2701 & 3 & 3 & 5 & 3 & 6 & 1 & (3) & 1 & 1 & 1 & 3 & 0 \\
\hline 2713 & 1 & 1 & 1 & 2 & 2 & 1 & (9) & 1 & 3 & 1 & 9 & * \\
\hline 2737 & 2 & 2 & 2 & 2 & 3 & 1 & (3) & 1 & 1 & 1 & 3 & 0 \\
\hline 2743 & 2 & 2 & 3 & 2 & 4 & 1 & (3) & 1 & 1 & 1 & 3 & 0 \\
\hline 2794 & 2 & 2 & 3 & 3 & 3 & 2 & (9) & 1 & 1 & 3 & 9 & $*$ \\
\hline 2917 & 3 & 3 & 3 & 4 & 4 & 3 & $(3,3)$ & 3 & 3 & 3 & 9 & $*$ \\
\hline 2971 & 1 & 1 & 1 & 2 & 2 & 1 & (9) & 1 & 3 & 3 & 9 & 0 \\
\hline 3001 & 2 & 2 & 2 & 2 & 2 & 2 & (3) & 1 & 1 & 3 & 9 & 0 \\
\hline 3094 & 2 & 2 & 2 & 2 & 2 & 2 & (3) & 1 & 1 & 3 & 9 & 0 \\
\hline 3133 & 3 & 3 & 5 & 3 & 6 & 1 & (3) & 1 & 1 & 1 & 3 & 0 \\
\hline 3190 & 2 & 2 & 2 & 2 & 3 & 1 & (3) & 1 & 1 & 1 & 3 & 0 \\
\hline 3199 & 2 & 2 & 2 & 2 & 3 & 1 & (3) & 1 & 1 & 1 & 3 & 0 \\
\hline 3226 & 2 & 2 & 2 & 2 & 3 & 1 & (9) & 1 & 1 & 1 & 3 & 0 \\
\hline 3235 & 2 & 2 & 2 & 2 & 3 & 1 & (9) & 1 & 1 & 1 & 3 & 0 \\
\hline 3277 & 2 & 2 & 2 & 2 & 3 & 1 & $(27)$ & 1 & 1 & 1 & 3 & 0 \\
\hline 3355 & 2 & 2 & 2 & 2 & 2 & 3 & $(3)$ & 1 & 1 & 3 & 9 & 0 \\
\hline 3391 & 2 & 2 & 4 & 2 & 5 & 2 & $(3,3)$ & 3 & 3 & 3 & 9 & 0 \\
\hline 3469 & 2 & 2 & 2 & 3 & 3 & 2 & (3) & 1 & 1 & 1 & 9 & * \\
\hline 3490 & 2 & 2 & 2 & 3 & 3 & 1 & (9) & 1 & 1 & 1 & 3 & * \\
\hline 3571 & 2 & 2 & 2 & 2 & 3 & 1 & (3) & 1 & 1 & 1 & 3 & 0 \\
\hline 3667 & 2 & 2 & 2 & 2 & 3 & 2 & $(3,3)$ & 3 & 3 & 3 & 9 & 0 \\
\hline 3673 & 2 & 2 & 4 & 2 & 5 & 1 & (3) & 1 & 1 & 1 & 3 & 0 \\
\hline 3739 & 2 & 2 & 2 & 3 & 3 & 1 & (3) & 1 & 3 & 1 & 9 & * \\
\hline 3781 & 2 & 2 & 2 & 2 & 3 & 1 & (9) & 1 & 1 & 1 & 3 & 0 \\
\hline 3787 & 2 & 2 & 2 & 2 & 2 & 2 & (3) & 1 & 1 & 3 & 9 & 0 \\
\hline 3847 & 2 & 2 & 2 & 2 & 2 & 2 & (3) & 1 & 1 & 3 & 9 & 0 \\
\hline 3895 & 2 & 2 & 3 & 2 & 4 & 1 & (3) & 1 & 1 & 1 & 3 & 0 \\
\hline
\end{tabular}


Table 1 (cont.)

\begin{tabular}{|c|c|c|c|c|c|c|c|c|c|c|c|c|}
\hline$m$ & $n_{0}$ & $n_{1}$ & $n_{2}$ & $n_{0}^{(1)}$ & $n_{2}^{(1)}$ & $\lambda_{3}^{-}\left(k^{*}\right)$ & $A_{0}^{*-}$ & $\left|D_{0}\right|$ & $\left|A_{0}\right|$ & $\left|D_{1}\right|$ & $\left|A_{1}\right|$ & $\lambda_{3}(k)$ \\
\hline 3979 & 2 & 2 & 3 & 2 & 4 & 1 & (3) & 1 & 1 & 1 & 3 & 0 \\
\hline 3997 & 2 & 2 & 2 & 2 & 3 & 1 & (9) & 1 & 1 & 1 & 3 & 0 \\
\hline 4081 & 3 & 3 & 3 & 4 & 4 & 1 & (3) & 1 & 1 & 1 & 3 & $*$ \\
\hline 4099 & 2 & 2 & 2 & 2 & 3 & 2 & (3) & 1 & 1 & 1 & 27 & 0 \\
\hline 4207 & 2 & 2 & 2 & 2 & 3 & 1 & (9) & 1 & 1 & 1 & 3 & 0 \\
\hline 4210 & 2 & 2 & 2 & 2 & 3 & 1 & (9) & 1 & 1 & 1 & 3 & 0 \\
\hline 4222 & 2 & 2 & 2 & 2 & 2 & 2 & (3) & 1 & 1 & 3 & 9 & 0 \\
\hline 4237 & 2 & 2 & 2 & 2 & 3 & 1 & (3) & 1 & 1 & 1 & 3 & 0 \\
\hline 4279 & 3 & 3 & 3 & 3 & 3 & 2 & $(3,3)$ & 3 & 3 & 9 & 27 & $*$ \\
\hline 4447 & 2 & 2 & 2 & 2 & 3 & 2 & (3) & 1 & 1 & 1 & 9 & 0 \\
\hline 4471 & 1 & 1 & 1 & 2 & 2 & 1 & (3) & 1 & 3 & 3 & 9 & 0 \\
\hline 4498 & 2 & 2 & 2 & 2 & 3 & 1 & (3) & 1 & 1 & 1 & 3 & 0 \\
\hline 4519 & 2 & 2 & 2 & 2 & 3 & 1 & (3) & 1 & 1 & 1 & 3 & 0 \\
\hline 4603 & 2 & 2 & 2 & 2 & 3 & 1 & (27) & 1 & 1 & 1 & 3 & 0 \\
\hline 4615 & 2 & 2 & 3 & 2 & 4 & 1 & (3) & 1 & 1 & 1 & 3 & 0 \\
\hline 4618 & 2 & 2 & 4 & 2 & 5 & 1 & (3) & 1 & 1 & 1 & 3 & 0 \\
\hline 4651 & 1 & 2 & 2 & 2 & 3 & 1 & (3) & 1 & 3 & 3 & 9 & 0 \\
\hline 4654 & 2 & 2 & 2 & 3 & 3 & 1 & (3) & 1 & 1 & 1 & 3 & $*$ \\
\hline 4681 & 2 & 2 & 2 & 2 & 3 & 1 & (3) & 1 & 1 & 1 & 3 & 0 \\
\hline 4687 & 2 & 2 & 2 & 2 & 2 & 3 & (3) & 1 & 1 & 3 & 9 & 0 \\
\hline 4711 & 2 & 2 & 2 & 2 & 3 & 1 & (3) & 1 & 1 & 1 & 3 & 0 \\
\hline 4741 & 2 & 2 & 3 & 3 & 3 & 3 & (9) & 1 & 1 & 3 & 9 & $*$ \\
\hline 4789 & 2 & 2 & 2 & 3 & 3 & 1 & (9) & 1 & 1 & 1 & 3 & $*$ \\
\hline 4837 & 2 & 2 & 2 & 2 & 2 & 3 & (3) & 1 & 1 & 3 & 9 & 0 \\
\hline 4867 & 2 & 2 & 2 & 2 & 3 & 1 & (3) & 1 & 1 & 1 & 3 & 0 \\
\hline 4870 & 2 & 2 & 2 & 2 & 3 & 1 & (9) & 1 & 1 & 1 & 3 & 0 \\
\hline 4954 & 1 & 1 & 1 & 2 & 2 & 1 & (3) & 3 & 9 & 9 & 27 & 0 \\
\hline 4963 & 2 & 2 & 3 & 2 & 4 & 1 & (3) & 1 & 1 & 1 & 3 & 0 \\
\hline 5005 & 2 & 2 & 2 & 2 & 2 & 2 & (3) & 1 & 1 & 3 & 9 & 0 \\
\hline 5062 & 3 & 3 & 3 & 3 & 4 & 1 & (3) & 1 & 1 & 1 & 3 & 0 \\
\hline 5083 & 2 & 2 & 2 & 2 & 3 & 1 & (3) & 1 & 1 & 1 & 3 & 0 \\
\hline 5113 & 2 & 2 & 2 & 2 & 3 & 1 & (3) & 1 & 1 & 1 & 3 & 0 \\
\hline 5149 & 2 & 2 & 2 & 2 & 3 & 1 & (9) & 1 & 1 & 1 & 3 & 0 \\
\hline 5161 & 2 & 2 & 2 & 2 & 2 & 2 & (3) & 1 & 1 & 3 & 9 & 0 \\
\hline 5182 & 2 & 2 & 2 & 2 & 3 & 1 & (3) & 1 & 1 & 1 & 3 & 0 \\
\hline 5185 & 2 & 2 & 2 & 3 & 3 & 1 & (3) & 1 & 1 & 1 & 3 & $*$ \\
\hline 5365 & 2 & 2 & 2 & 2 & 2 & 2 & (3) & 1 & 1 & 3 & 9 & 0 \\
\hline 5386 & 2 & 2 & 2 & 2 & 2 & 2 & (3) & 1 & 1 & 3 & 9 & 0 \\
\hline 5407 & 2 & 2 & 2 & 2 & 2 & 2 & (3) & 1 & 1 & 3 & 27 & 0 \\
\hline 5437 & 2 & 2 & 2 & 2 & 2 & 2 & (3) & 1 & 1 & 3 & 9 & 0 \\
\hline 5458 & 2 & 2 & 2 & 2 & 2 & 2 & (3) & 1 & 1 & 3 & 9 & 0 \\
\hline 5494 & 2 & 2 & 2 & 2 & 3 & 1 & (3) & 1 & 1 & 1 & 3 & 0 \\
\hline 5530 & 2 & 2 & 2 & 3 & 3 & 2 & (3) & 1 & 1 & 1 & 9 & $*$ \\
\hline 5533 & 2 & 2 & 3 & 3 & 3 & 2 & (9) & 1 & 1 & 3 & 9 & $*$ \\
\hline 5611 & 3 & 3 & 3 & 3 & 3 & 3 & (9) & 1 & 1 & 3 & 9 & $*$ \\
\hline 5617 & 2 & 2 & 2 & 2 & 3 & 1 & (9) & 1 & 1 & 1 & 3 & 0 \\
\hline
\end{tabular}


Table 1 (cont.)

\begin{tabular}{|c|c|c|c|c|c|c|c|c|c|c|c|c|}
\hline$m$ & $n_{0}$ & $n_{1}$ & $n_{2}$ & $n_{0}^{(1)}$ & $n_{2}^{(1)}$ & $\lambda_{3}^{-}\left(k^{*}\right)$ & $A_{0}^{*-}$ & $\left|D_{0}\right|$ & $\left|A_{0}\right|$ & $\left|D_{1}\right|$ & $\left|A_{1}\right|$ & $\lambda_{3}(k)$ \\
\hline 5647 & 2 & 2 & 3 & 2 & 4 & 1 & (3) & 1 & 1 & 1 & 3 & 0 \\
\hline 5749 & 2 & 2 & 2 & 2 & 3 & 1 & (27) & 1 & 1 & 1 & 3 & 0 \\
\hline 5902 & 2 & 2 & 2 & 2 & 3 & 1 & (9) & 1 & 1 & 1 & 3 & 0 \\
\hline 5938 & 1 & 1 & 1 & 2 & 2 & 1 & (27) & 1 & 3 & 1 & 9 & $*$ \\
\hline 5971 & 2 & 2 & 3 & 3 & 3 & 3 & (9) & 1 & 1 & 3 & 27 & $*$ \\
\hline 6001 & 2 & 2 & 2 & 2 & 3 & 1 & (9) & 1 & 1 & 1 & 3 & 0 \\
\hline 6169 & 2 & 2 & 2 & 3 & 3 & 1 & (3) & 1 & 1 & 1 & 3 & * \\
\hline 6187 & 2 & 2 & 2 & 3 & 3 & 3 & (3) & 1 & 1 & 1 & 9 & $*$ \\
\hline 6202 & 2 & 2 & 2 & 3 & 3 & 1 & (3) & 1 & 1 & 1 & 3 & $*$ \\
\hline 6238 & 1 & 1 & 1 & 2 & 2 & 1 & (3) & 1 & 3 & 3 & 9 & 0 \\
\hline 6271 & 2 & 2 & 2 & 3 & 3 & 1 & (3) & 1 & 1 & 1 & 3 & $*$ \\
\hline 6286 & 2 & 2 & 2 & 3 & 3 & 1 & (9) & 1 & 1 & 1 & 3 & $*$ \\
\hline 6295 & 2 & 2 & 2 & 2 & 2 & 2 & (3) & 1 & 1 & 3 & 9 & 0 \\
\hline 6355 & 2 & 2 & 2 & 2 & 3 & 1 & (3) & 1 & 1 & 1 & 3 & 0 \\
\hline 6403 & 2 & 2 & 2 & 2 & 3 & 1 & (9) & 1 & 1 & 1 & 3 & 0 \\
\hline 6430 & 2 & 2 & 2 & 2 & 2 & 3 & (3) & 1 & 1 & 3 & 9 & 0 \\
\hline 6451 & 2 & 2 & 2 & 2 & 2 & 2 & (3) & 1 & 1 & 3 & 27 & 0 \\
\hline 6502 & 2 & 2 & 2 & 2 & 3 & 1 & (9) & 1 & 1 & 1 & 3 & 0 \\
\hline 6559 & 2 & 2 & 4 & 3 & 4 & 2 & $(3,3)$ & 9 & 9 & 27 & 81 & $*$ \\
\hline 6601 & 1 & 1 & 1 & 2 & 2 & 2 & (3) & 1 & 3 & 3 & 9 & 0 \\
\hline 6691 & 2 & 2 & 2 & 2 & 3 & 1 & (3) & 1 & 1 & 1 & 3 & 0 \\
\hline 6730 & 2 & 2 & 2 & 2 & 3 & 1 & (9) & 1 & 1 & 1 & 3 & 0 \\
\hline 6799 & 2 & 2 & 2 & 2 & 2 & 2 & (3) & 1 & 1 & 3 & 9 & 0 \\
\hline 6871 & 2 & 2 & 2 & 3 & 3 & 1 & $(27)$ & 1 & 1 & 1 & 3 & $*$ \\
\hline 6901 & 1 & 1 & 1 & 2 & 2 & 2 & (3) & 1 & 3 & 3 & 9 & 0 \\
\hline 6907 & 2 & 2 & 2 & 2 & 3 & 1 & (3) & 1 & 1 & 1 & 3 & 0 \\
\hline 6934 & 2 & 2 & 2 & 3 & 3 & 1 & (9) & 1 & 1 & 1 & 3 & $*$ \\
\hline 6949 & 2 & 2 & 2 & 2 & 2 & 2 & (3) & 1 & 1 & 3 & 9 & 0 \\
\hline 6955 & 3 & 3 & 4 & 3 & 5 & 1 & (3) & 1 & 1 & 1 & 3 & 0 \\
\hline 7006 & 3 & 3 & 3 & 3 & 4 & 3 & $(3,3)$ & 3 & 3 & 3 & 9 & * \\
\hline 7051 & 2 & 2 & 2 & 2 & 3 & 1 & (9) & 1 & 1 & 1 & 3 & 0 \\
\hline 7078 & 2 & 2 & 4 & 2 & 5 & 1 & (3) & 1 & 1 & 1 & 3 & 0 \\
\hline 7234 & 1 & 1 & 1 & 2 & 2 & 2 & (3) & 1 & 3 & 3 & 9 & 0 \\
\hline 7246 & 2 & 2 & 3 & 2 & 4 & 2 & (9) & 1 & 1 & 1 & 9 & 0 \\
\hline 7294 & 2 & 2 & 2 & 2 & 3 & 1 & (9) & 1 & 1 & 1 & 3 & 0 \\
\hline 7303 & 2 & 2 & 2 & 2 & 2 & 3 & (3) & 1 & 1 & 3 & 9 & 0 \\
\hline 7309 & 2 & 2 & 2 & 3 & 3 & 1 & (9) & 1 & 1 & 1 & 3 & $*$ \\
\hline 7315 & 2 & 2 & 2 & 2 & 3 & 2 & (3) & 1 & 1 & 1 & 9 & 0 \\
\hline 7321 & 2 & 2 & 2 & 3 & 3 & 1 & (3) & 1 & 1 & 1 & 3 & * \\
\hline 7387 & 1 & 1 & 1 & 2 & 2 & 1 & (9) & 1 & 3 & 3 & 9 & 0 \\
\hline 7429 & 2 & 2 & 3 & 3 & 3 & 2 & (9) & 1 & 1 & 3 & 9 & * \\
\hline 7465 & 3 & 3 & 3 & 3 & 4 & 2 & $(3,3)$ & 9 & 9 & 9 & 27 & $*$ \\
\hline 7522 & 2 & 2 & 2 & 2 & 3 & 1 & (3) & 1 & 1 & 1 & 3 & 0 \\
\hline 7582 & 2 & 2 & 2 & 3 & 3 & 1 & (3) & 1 & 1 & 1 & 3 & * \\
\hline 7603 & 2 & 2 & 2 & 2 & 3 & 1 & (27) & 1 & 1 & 1 & 3 & 0 \\
\hline 7621 & 2 & 2 & 2 & 2 & 3 & 1 & $(3)$ & 1 & 1 & 1 & 3 & 0 \\
\hline
\end{tabular}


Table 1 (cont.)

\begin{tabular}{|c|c|c|c|c|c|c|c|c|c|c|c|c|}
\hline$m$ & $n_{0}$ & $n_{1}$ & $n_{2}$ & $n_{0}^{(1)}$ & $n_{2}^{(1)}$ & $\lambda_{3}^{-}\left(k^{*}\right)$ & $A_{0}^{*-}$ & $\left|D_{0}\right|$ & $\left|A_{0}\right|$ & $\left|D_{1}\right|$ & $\left|A_{1}\right|$ & $\lambda_{3}(k)$ \\
\hline 7633 & 2 & 2 & 2 & 2 & 3 & 1 & (9) & 1 & 1 & 1 & 3 & 0 \\
\hline 7639 & 1 & 1 & 1 & 2 & 2 & 1 & (3) & 1 & 3 & 3 & 9 & 0 \\
\hline 7642 & 2 & 2 & 3 & 3 & 3 & 2 & (27) & 1 & 1 & 3 & 9 & $*$ \\
\hline 7705 & 2 & 2 & 2 & 2 & 2 & 2 & (3) & 1 & 1 & 3 & 9 & 0 \\
\hline 7711 & 1 & 2 & 2 & 2 & 3 & 1 & (3) & 1 & 3 & 3 & 9 & 0 \\
\hline 7726 & 2 & 2 & 2 & 2 & 3 & 3 & $(3,3)$ & 1 & 3 & 1 & 81 & $*$ \\
\hline 7753 & 1 & 2 & 2 & 2 & 3 & 2 & (9) & 1 & 3 & 3 & 27 & 0 \\
\hline 7906 & 2 & 2 & 2 & 2 & 3 & 1 & (9) & 1 & 1 & 1 & 3 & 0 \\
\hline 7951 & 2 & 2 & 3 & 2 & 4 & 1 & (3) & 1 & 1 & 1 & 3 & 0 \\
\hline 7954 & 2 & 2 & 3 & 2 & 4 & 2 & $(3,3)$ & 3 & 3 & 3 & 9 & 0 \\
\hline 7957 & 2 & 2 & 2 & 3 & 3 & 1 & (3) & 1 & 1 & 1 & 3 & $*$ \\
\hline 7969 & 3 & 3 & 3 & 3 & 4 & 1 & (3) & 1 & 1 & 1 & 3 & 0 \\
\hline 7978 & 2 & 2 & 2 & 2 & 2 & 2 & (3) & 1 & 1 & 3 & 9 & 0 \\
\hline 8011 & 2 & 2 & 2 & 2 & 3 & 1 & (3) & 1 & 1 & 1 & 3 & 0 \\
\hline 8017 & 1 & 1 & 1 & 2 & 2 & 1 & (3) & 1 & 3 & 1 & 9 & $*$ \\
\hline 8095 & 2 & 2 & 2 & 2 & 3 & 1 & (3) & 1 & 1 & 1 & 3 & 0 \\
\hline 8101 & 2 & 2 & 2 & 3 & 3 & 1 & (3) & 1 & 1 & 1 & 3 & $*$ \\
\hline 8137 & 2 & 2 & 2 & 2 & 3 & 2 & (3) & 1 & 1 & 1 & 27 & 0 \\
\hline 8155 & 2 & 2 & 2 & 3 & 3 & 1 & (3) & 1 & 1 & 1 & 3 & $*$ \\
\hline 8194 & 2 & 2 & 2 & 2 & 2 & 4 & (3) & 1 & 1 & 3 & 9 & 0 \\
\hline 8203 & 2 & 2 & 2 & 2 & 3 & 1 & (3) & 1 & 1 & 1 & 3 & 0 \\
\hline 8209 & 2 & 2 & 2 & 2 & 2 & 2 & (3) & 1 & 1 & 3 & 9 & 0 \\
\hline 8245 & 2 & 2 & 2 & 2 & 3 & 1 & (3) & 1 & 1 & 1 & 3 & 0 \\
\hline 8365 & 2 & 2 & 2 & 2 & 3 & 1 & (9) & 1 & 1 & 1 & 3 & 0 \\
\hline 8374 & 2 & 2 & 3 & 2 & 4 & 3 & $(3,3)$ & 3 & 3 & 3 & 27 & 0 \\
\hline 8422 & 2 & 2 & 2 & 2 & 3 & 1 & (3) & 1 & 1 & 1 & 3 & 0 \\
\hline 8545 & 1 & 1 & 1 & 2 & 2 & 1 & (9) & 1 & 3 & 3 & 9 & 0 \\
\hline 8569 & 2 & 2 & 2 & 3 & 3 & 1 & (3) & 1 & 1 & 1 & 3 & $*$ \\
\hline 8599 & 2 & 2 & 2 & 2 & 3 & 1 & (3) & 1 & 1 & 1 & 3 & 0 \\
\hline 8626 & 2 & 2 & 2 & 2 & 3 & 1 & (3) & 1 & 1 & 1 & 3 & 0 \\
\hline 8713 & 2 & 2 & 3 & 2 & 4 & 2 & $(3,3)$ & 3 & 3 & 3 & 9 & 0 \\
\hline 8755 & 2 & 2 & 2 & 2 & 3 & 1 & (3) & 1 & 1 & 1 & 3 & 0 \\
\hline 8758 & 2 & 2 & 2 & 2 & 2 & 4 & (3) & 1 & 1 & 3 & 9 & 0 \\
\hline 8782 & 1 & 1 & 1 & 2 & 2 & 1 & (9) & 1 & 3 & 1 & 9 & $*$ \\
\hline 8785 & 2 & 2 & 3 & 2 & 4 & 1 & (3) & 1 & 1 & 1 & 3 & 0 \\
\hline 8809 & 2 & 2 & 4 & 2 & 5 & 1 & (3) & 1 & 1 & 1 & 3 & 0 \\
\hline 8821 & 2 & 2 & 4 & 2 & 5 & 1 & (3) & 1 & 1 & 1 & 3 & 0 \\
\hline 8854 & 1 & 1 & 1 & 2 & 2 & 2 & (3) & 1 & 3 & 3 & 9 & 0 \\
\hline 8863 & 1 & 2 & 2 & 2 & 3 & 1 & (3) & 1 & 3 & 3 & 9 & 0 \\
\hline 8893 & 2 & 2 & 2 & 2 & 2 & 2 & (3) & 1 & 1 & 3 & 9 & 0 \\
\hline 8965 & 3 & 3 & 3 & 3 & 4 & 1 & (3) & 1 & 1 & 1 & 3 & 0 \\
\hline 9019 & 2 & 2 & 2 & 2 & 3 & 1 & (9) & 1 & 1 & 1 & 3 & 0 \\
\hline 9034 & 1 & 1 & 1 & 2 & 2 & 1 & (27) & 1 & 3 & 3 & 9 & 0 \\
\hline 9058 & 2 & 2 & 2 & 3 & 3 & 1 & (9) & 1 & 1 & 1 & 3 & $*$ \\
\hline 9097 & 2 & 2 & 2 & 2 & 2 & 2 & (3) & 1 & 1 & 3 & 27 & 0 \\
\hline 9103 & 2 & 2 & 2 & 2 & 3 & 1 & (27) & 1 & 1 & 1 & 3 & 0 \\
\hline
\end{tabular}


Table 1 (cont.)

\begin{tabular}{|c|c|c|c|c|c|c|r|c|c|c|c|c|}
\hline$m$ & $n_{0}$ & $n_{1}$ & $n_{2}$ & $n_{0}^{(1)}$ & $n_{2}^{(1)}$ & $\lambda_{3}^{-}\left(k^{*}\right)$ & $A_{0}^{*-}$ & $\left|D_{0}\right|$ & $\left|A_{0}\right|$ & $\left|D_{1}\right|$ & $\left|A_{1}\right|$ & $\lambda_{3}(k)$ \\
\hline 9115 & 2 & 2 & 3 & 2 & 4 & 1 & $(3)$ & 1 & 1 & 1 & 3 & 0 \\
9145 & 2 & 2 & 2 & 2 & 3 & 1 & $(3)$ & 1 & 1 & 1 & 3 & 0 \\
9202 & 2 & 2 & 2 & 2 & 3 & 1 & $(3)$ & 1 & 1 & 1 & 3 & 0 \\
9274 & 4 & 4 & 5 & 4 & 6 & 1 & $(3)$ & 1 & 1 & 1 & 3 & 0 \\
9427 & 2 & 2 & 2 & 2 & 3 & 3 & $(3)$ & 1 & 1 & 1 & 9 & 0 \\
9463 & 2 & 2 & 3 & 2 & 4 & 1 & $(3)$ & 1 & 1 & 1 & 3 & 0 \\
9586 & 1 & 1 & 1 & 2 & 2 & 3 & $(3)$ & 1 & 3 & 3 & 9 & 0 \\
9634 & 3 & 3 & 4 & 3 & 5 & 2 & $(9,3)$ & 3 & 3 & 3 & 9 & $*$ \\
9679 & 4 & 4 & 6 & 4 & 7 & 1 & $(3)$ & 1 & 1 & 1 & 3 & 0 \\
9691 & 2 & 2 & 3 & 3 & 3 & 2 & $(9)$ & 1 & 1 & 3 & 9 & $*$ \\
9754 & 2 & 2 & 4 & 2 & 5 & 1 & $(3)$ & 1 & 1 & 1 & 3 & 0 \\
9766 & 1 & 1 & 1 & 2 & 2 & 1 & $(3)$ & 1 & 3 & 3 & 9 & 0 \\
9790 & 2 & 2 & 2 & 2 & 3 & 4 & $(3,3)$ & 3 & 3 & 3 & 27 & 0 \\
9814 & 4 & 4 & 4 & 5 & 5 & 1 & $(3)$ & 1 & 1 & 1 & 3 & $*$ \\
9895 & 3 & 3 & 3 & 3 & 4 & 1 & $(3)$ & 1 & 1 & 1 & 3 & 0 \\
\hline
\end{tabular}

\section{References}

[1] A. Candiotti, Computations of Iwasawa invariants and $K_{2}$, Compositio Math. 29 (1974), 89-111.

[2] B. Ferrero and L. C. W a shington, The Iwasawa invariant $\mu_{p}$ vanishes for abelian number fields, Ann. of Math. 109 (1979), 377-395.

[3] T. Fukuda, Iwasawa $\lambda$-invariants of certain real quadratic fields, Proc. Japan Acad. 65A (1989), 260-262.

[4] -, Iwasawa $\lambda$-invariants of imaginary quadratic fields, J. College Industrial Technology Nihon Univ. 27 (1994), 35-88. (Corrigendum; to appear ibid.)

[5] T. Fukuda and K. Komatsu, On the $\lambda$ invariants of $\mathbb{Z}_{p}$-extensions of real quadratic fields, J. Number Theory 23 (1986), 238-242.

[6] - - - On $\mathbb{Z}_{p}$-extensions of real quadratic fields, J. Math. Soc. Japan 38 (1986), 95-102.

[7] T. F ukuda, K. Kom ats u and H. W ad a, A remark on the $\lambda$-invariants of real quadratic fields, Proc. Japan Acad. 62A (1986), 318-319.

[8] R. Green berg, On the Iwasawa invariants of totally real number fields, Amer. J. Math. 98 (1976), 263-284.

[9] -, On p-adic L-functions and cyclotomic fields II, Nagoya Math. J. 67 (1977), 139-158.

[10] K. Iw as awa, On $\mathbb{Z}_{l}$-extensions of algebraic number fields, Ann. of Math. 98 (1973), 246-326.

[11] S. Mäki, The determination of units in real cyclic sextic fields, Lecture Notes in Math. 797, Springer, Berlin, 1980.

[12] H. T a y a, On the Iwasawa $\lambda$-invariants of real quadratic fields, Tokyo J. Math. 16 (1993), 121-130.

[13] - Computation of $\mathbb{Z}_{3}$-invariants of real quadratic fields, preprint series, Waseda Univ. Technical Report No. 93-13, 1993. 
[14] H. W a d a and M. S a ito, A table of ideal class groups of imaginary quadratic fields, Sophia Kôkyuroku in Math. 28, Depart. of Math., Sophia Univ. Tokyo, 1988.

[15] L. C. W a s h ing to n, Introduction to Cyclotomic Fields, Graduate Texts in Math. 83, Springer, New York, 1982.

[16] H. Yok oi, On the class number of a relatively cyclic number field, Nagoya Math. J. 29 (1967), 31-44.

DEPARTMENT OF MATHEMATICS COLLEGE OF INDUSTRIAL TECHNOLOGY NIHON UNIVERSITY

2-11-1, SHIN-EI NARASHINO-SHI

CHIBA, 275 JAPAN

E-mail: FUKUDA@MATH.CIT.NIHON-U.AC.JP
DEPARTMENT OF MATHEMATICS SCHOOL OF SCIENCE AND ENGINEERING WASEDA UNIVERSITY 3-4-1, OKUBO SHINJUKU-KU TOKYO, 169 JAPAN E-mail: TAYA@CFI.WASEDA.AC.JP

Received on 28.3.1994

and in revised form on 5.6.1994 